\title{
The time series regression analysis in evaluating the economic impact of COVID-19 cases in Indonesia
}

\author{
Utriweni Mukhaiyar*, Devina Widyanti and Sandy Vantika \\ Statistics Research Division, Faculty of Mathematics and Natural Sciences, Institut Teknologi Bandung, Bandung, \\ Indonesia
}

\begin{abstract}
This study aims to determine the impact of COVID-19 cases in Indonesia on the USD/IDR exchange rate using the Transfer Function Model and Vector Autoregressive Moving-Average with Exogenous Regressors (VARMAX) Model. This paper uses daily data on the COVID-19 case in Indonesia, the USD/IDR exchange rate, and the IDX Composite period from 1 March to 29 June 2020. The analysis shows: (1) the higher the increase of the number of COVID-19 cases in Indonesia will significantly weaken the USD/IDR exchange rate, (2) an increase of $1 \%$ in the number of COVID-19 cases in Indonesia six days ago will weaken the USD/IDR exchange rate by $0.003 \%$, (3) an increase of $1 \%$ in the number of COVID-19 cases in Indonesia seven days ago will weaken the USD/IDR exchange rate by $0.17 \%$, and (4) an increase of $1 \%$ in the number of COVID-19 cases in Indonesia eight days ago will weaken the USD/IDR exchange rate by $0.24 \%$.
\end{abstract}

Keywords: Transfer function model, VARMAX model, COVID-19, the USD/IDR exchange rate

\section{Introduction}

The world economic situation has deteriorated due to the increasing number of countries affected by the COVID-19 virus in the world such as America, Italy, and Spain. Trends in the number of patients and those who have died have not shown any decline even though the number of people who have recovered has increased compared to those who died. The impact of COVID-19 caused the exchange rates of several countries to fluctuate, for instance, Thai Baht, Mexican Peso, and Russian Ruble. The impact of COVID-19 hit Indonesian financial markets as well. Finance Minister Sri Mulyani said that Indonesia was quite affected by the spread of COVID-19, not only in the human health sector but also had disrupted the health of the global economy. The impact of this virus has resulted in various economic aspects; the stock exchange index fell, the USD/IDR exchange rate weakened drastically, MSME entrepreneurs struggled. Prior to the COVID-19 pandemic, the USD/IDR exchange rate was in the range of Rp $14,000.00$ and during the pandemic, the Rupiah was briefly pressured down to the level of $\mathrm{Rp} 16,500.00$. This COVID-19 pandemic caused panic in the global market, so investors withdrew their funds from Indonesia and placed them in a safe haven. This makes the USD/IDR exchange rate continue to weaken. The COVID-19 not only affects the USD/IDR exchange rate, but also has an impact on the decline in the IDX Composite to Rp 3,900.00. In addition, several countries also experienced the same thing, for example, SPI Singapore and SET Thailand. Therefore, it is interesting to see the impact of COVID-19 on the USD/IDR exchange rate and IDX composite.

The involvement of causal (external) factors into a time series can be viewed as part of a time series regression. Cryer and Chan (2006) consider it as intervention or outlier effects. If the external factor be the input and the response

\footnotetext{
${ }^{*}$ Corresponding author: Utriweni Mukhaiyar, Faculty of Mathematics and Natural Sciences, Institut Teknologi Bandung, Bandung, Indonesia. E-mail: utriweni@math.itb.ac.id.
} 
be the output series, then it can be a transfer function model (Box et al., 2016). Beside transfer function models, other name that are occasionally found in the related literature are distributed lag models (Lutkepohl, 2005). Many researches were conducted to model the cause-effect relationship among two or more time series. Nicholson et al. (2015) introduced VARX-L model to overcome the high-dimensional macroeconomic problem and Wei (2019) did a quantitative structural analysis to evaluate oil price shocks, economic policy uncertainty and China's trade. Mukhaiyar et al. (2019) modeled the Dengue Fever Cases by considering the oulier factor using GSTAR $(1 ; 1)$ model meanwhile with the similar model Masteriana et al. (2019) used for analysis the Begal Criminal cases. By considering exogenous variable (average of monthly temperature) and outliers, Mukhaiyar et al. (2020) predict the monthly cases of dengue fever while Huda et al. (2020) used ADL model with outlier factor to do the prediction. Hasan et al. (2021) modeled the effect of COVID-19 pandemic on global economic, stock market, and the energy sector based on SVAR model, meanwhile Ganegoda et al. (2021) evaluated the interrelationship between daily COVID-19 cases and weather variables through temporal and spatial auto-correlation, and clustering-integrated panel regression. Saikhu et al. (2021) used ARIMA, bivariate and multivariate transfer function to predict COVID-19 cases. The effect of spatial dependence through railroad passenger's mobility was considered by Pasaribu et al. (2021) to model the COVID-19 growth cases in Java island. More applications can be found in oscillation damping controllers of the power system (Liu et al., 2015), finance (Apriliandra et al., 2016), and residential building cost (Zhao et al., 2019) In this paper, the multivariate transfer function is explored to model the impact of the COVID-19 cases on the USD/IDR exchange rate and IDX composite, especially in the early days of the pandemic in Indonesia.

\section{Methods}

\subsection{The transfer function model}

The transfer function model is a model describing the future predictive value of a time series (output series $\left\{Y_{t}\right\}$ ) based on the past values of the series itself and one or more time-series related to the output series (input series $\left.\left\{X_{t}\right\}\right)$. This model involves two or more variables consisting of the output variable $\left\{Y_{t}\right\}$, the variable input $\left\{X_{t}\right\}$, and another input called residual $\left\{N_{t}\right\}$ that has a relationship causality.

Assume $\left\{X_{t}\right\}$ and $\left\{Y_{t}\right\}$ are stationary series. In a single input case, $\left\{Y_{t}\right\}$ and $\left\{X_{t}\right\}$ can be written in the form:

$$
Y_{t}=v(B) X_{t}+N_{t}
$$

where $v(B)=\frac{\omega(B) B^{b}}{\delta(B)}$ is a transfer function where $\omega(B)=\omega_{0}-\omega_{1} B-\ldots-\omega_{s} B^{s}, \delta(B)=1-\delta_{1} B-\ldots-\delta_{r} B^{r}$, for $B$ is a backshift operator which is defined as $B^{\ell} X_{t}=X_{\ell-1}$. The $b$ is a delay parameter which shows the time lag when the input series starts to affect the output series, the $r$ indicates the pattern of the impulse response weights, and the $s$ shows the pattern of decay for impulse response weight begins. The $\left\{N_{t}\right\}$ is the total disturbance sequence (noise series) that affects $\left\{Y_{t}\right\}$ which is independent with $\left\{X_{t}\right\}$. The input series $\left\{X_{t}\right\}$ and noise series $\left\{N_{t}\right\}$ are assumed to follow the ARIMA model. Then $(b, r, s)$ is the main order in determining the best transfer function model. Since noise series follows the ARIMA Model, then the transfer function model can be written as (Wei, 2006):

$$
\begin{aligned}
Y_{t} & =v(B) X_{t}+N_{t} \\
& =\frac{\omega(B)}{\delta(B)} B^{b} X_{t}+\frac{\theta(B)}{\phi(B)} a_{t} \\
& =\frac{\omega(B)}{\delta(B)} X_{t-b}+\frac{\theta(B)}{\phi(B)} a_{t}
\end{aligned}
$$

where $\theta(B)=1-\theta_{1} B-\theta_{2} B^{2}-\ldots-\theta_{q} B^{q}$ and $\phi(B)=1-\phi_{B}-\phi_{2} B^{2}-\ldots-\phi_{p} B^{p}$. The VARMAX Model is a multivariate version of the transfer function model. Uniquely, the transfer function model is the case specifically from the VARMA Model.

\subsection{The VARMAX model}

According to Wei (2006), the VARMA Model $(p, q)$ can generally be stated in equation as follows:

$$
\boldsymbol{\Phi}_{p}(B) \boldsymbol{Y}_{t}=\boldsymbol{\Theta}_{q}(B) \boldsymbol{a}_{t}
$$

where $\boldsymbol{\Phi}_{p}(B)=\mathrm{I}_{k}-\boldsymbol{\Phi}_{1} B-\ldots-\boldsymbol{\Phi}_{p} B^{p}$ and $\boldsymbol{\Theta}_{q}(B)=\mathrm{I}_{k}-\boldsymbol{\Theta}_{1} B-\ldots-\boldsymbol{\Theta}_{q} B^{q}$. The $\boldsymbol{Y}_{t}$ is a vector multivariate 
time series, $\boldsymbol{\Phi}_{p}(B)$ is a $k \times k$ auto-regressive matrix polynomial of order $p$, and $\boldsymbol{\Theta}_{q}(B)$ is a $k \times k$ moving-average matrix polynomial of order $q$.

Then the VARMA $(1,1)$ SISO Model can be written in matrix form as follows:

$$
\left[\begin{array}{ll}
\Phi_{1 ; 1,1}(B) & \Phi_{1 ; 1,2}(B) \\
\Phi_{1 ; 2,1}(B) & \Phi_{1 ; 2,2}(B)
\end{array}\right]\left(\begin{array}{l}
Y_{1, t} \\
Y_{2, t}
\end{array}\right)=\left[\begin{array}{cc}
\Theta_{1 ; 1,1}(B) & \Theta_{1 ; 1,2}(B) \\
\Theta_{1 ; 2,1}(B) & \Theta_{1 ; 2,2}(B)
\end{array}\right]\left(\begin{array}{c}
a_{1, t} \\
a_{2, t}
\end{array}\right)
$$

Suppose $\Phi_{1 ; 1,2}(B)=\Theta_{1 ; 1,2}(B)=\Theta_{1 ; 2,1}(B)=0$. The VARMA (1,1) SISO Model Equation can be written as:

$$
\Phi_{1 ; 1,1}(B) Y_{1, t}=\Theta_{1 ; 1,1}(B) a_{1, t}
$$

and

$$
\Phi_{1 ; 2,2}(B) Y_{2, t}=-\Phi_{1 ; 2,1}(B) Y_{1 t}+\Theta_{1 ; 2,2}(B) a_{2, t}
$$

According to Eqs (2) and (3), the future value of $Y_{1, t}$ is only influenced by its past value but not affected by the past value of $Y_{2, t}$, while the future value of $Y_{2, t}$ is influenced by the future value then from $Y_{1, t}$ and $Y_{2, t}$.

This also shows that $Y_{1, t}$ results in $Y_{2, t}$ but $Y_{2, t}$ does not result in $Y_{1, t}$. So, $Y_{1, t}$ is called exogenous variable or it can also be called a predictor. Equation (3) is also called the ARMAX model or transfer function model with the output variable $Y_{2, t}$ and the input variable $Y_{1, t}$. Equation (3) can also be written as follows:

$$
Y_{2, t}=\Psi_{*}(B) Y_{1, t}+\Psi_{1 ; 2,2}(B) a_{2, t}
$$

where

$$
\Psi_{*}(B)=\frac{\Phi_{1 ; 2,1}(B)}{\Phi_{1 ; 2,2}(B)}
$$

and

$$
\Psi_{1 ; 2,2}(B)=\frac{\Theta_{1 ; 2,2}(B)}{\Phi_{1 ; 2,2}(B)}
$$

Equation (4) can be stated as:

$$
Y_{2, t}=\Psi_{*}(B) Y_{1, t}+N_{t}
$$

where $N_{t}$ is a noise series that follows the VARMA Model $\Psi_{1 ; 2,2}(B) N_{t}=\Theta_{1 ; 2,2}(B) a_{2, t}$. Hence, the ARMAX Model or transfer function model is a special case of the VARMA Model. Vector Autoregressive Moving-Average with Exogenous Regressors or VARMAX $(p, q, r)$ is a special case of the VARMA Model $(p, q)$ with the addition of exogenous/predictor variables. The VARMAX model is a multivariate version of the ARMAX model or the transfer function model (Widyanti, 2020). This model is suitable for multivariate time series without trends or seasonal components with the addition of exogenous variables. According to Spliid (1983), the VARMAX model $(p, q, r)$ can be written as follows:

$$
\boldsymbol{\Phi}_{p}(B) \boldsymbol{Y}_{t}=\gamma_{r}(B) \boldsymbol{X}_{t}+\boldsymbol{\Theta}_{q}(B) \boldsymbol{a}_{t}
$$

where, $\gamma_{r}(B)=\gamma_{0}+\gamma_{1} B+\ldots+\gamma_{r-1} B^{r-1}$. The $\boldsymbol{Y}_{t}$ is a $k \times 1$ multivariate vector time series, $\boldsymbol{X}_{t}$ is a $m \times 1$ predictor vector time series, $\boldsymbol{\Phi}_{p}(B)$ is a $k \times k$ autoregressive matrix polynomial of orde $p, \boldsymbol{\Theta}_{q}(B)$ is a $k \times k$ moving-average matrix polynomial of orde $\mathrm{q}, \boldsymbol{a}_{t}$ is a $k \times 1$ vector noise series, and $\gamma_{r}$ is a $k \times m$ matrix polynomial which its variance-covariance matrix $\sum$ of $\boldsymbol{a}_{t}$ is a positive definite.

Thus, the VARMAX Model $(p, q, r)$ Single Input Multi Output (SIMO) $(k=2)$ and 1 predictor $(m=1)$ can be written in the form of:

$$
\begin{aligned}
& \left(\begin{array}{c}
Y_{1, t} \\
Y_{2, t}
\end{array}\right)=\left[\begin{array}{ll}
\Phi_{1 ; 1,1} & \Phi_{1 ; 1,2} \\
\Phi_{1 ; 2,1} & \Phi_{1 ; 2,2}
\end{array}\right]\left(\begin{array}{c}
Y_{1, t-1} \\
Y_{2, t-1}
\end{array}\right)+\ldots+\left[\begin{array}{ll}
\Phi_{p ; 1,1} & \Phi_{p ; 1,2} \\
\Phi_{p ; 2,1} & \Phi_{p ; 2,2}
\end{array}\right]\left(\begin{array}{l}
Y_{1, t-p} \\
Y_{2, t-p}
\end{array}\right)+\left(\begin{array}{l}
\gamma_{0,1} \\
\gamma_{0,2}
\end{array}\right) X_{t} \\
& +\left(\begin{array}{c}
\gamma_{1,1} \\
\gamma_{1,2}
\end{array}\right) X_{t-1}+\ldots+\left(\begin{array}{c}
\gamma_{r-1,1} \\
\gamma_{r-1,2}
\end{array}\right) X_{t-(r-1)}+\left(\begin{array}{c}
a_{1, t} \\
a_{2, t}
\end{array}\right)+\left[\begin{array}{cc}
\Theta_{1 ; 1,1} & \Theta_{1 ; 1,2} \\
\Theta_{1 ; 2,1} & \Theta_{1 ; 2,2}
\end{array}\right]\left(\begin{array}{c}
a_{1, t-1} \\
a_{2, t-1}
\end{array}\right) \\
& +\ldots+\left[\begin{array}{cc}
\Theta_{q ; 1,1} & \Theta_{q ; 1,2} \\
\Theta_{q ; 2,1} & \Theta_{q ; 2,2}
\end{array}\right]\left(\begin{array}{c}
a_{1, t-q} \\
a_{2, t-q}
\end{array}\right)
\end{aligned}
$$




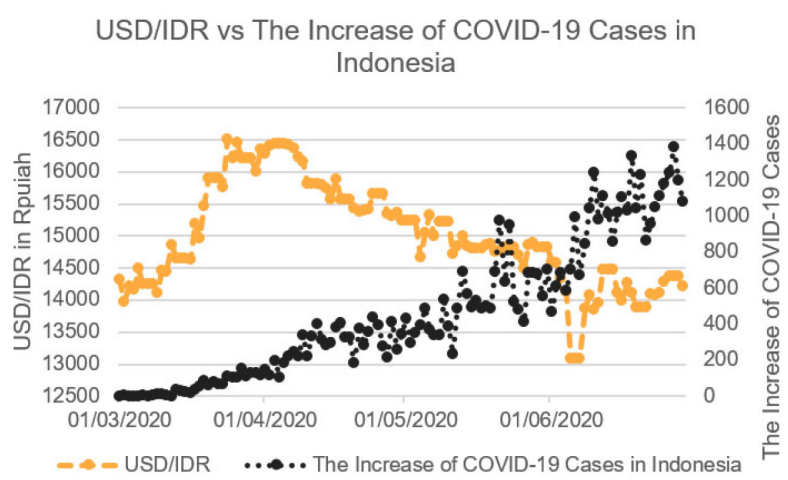

(a)
IDX Composite vs The Increase of COVID-19 Cases in Indonesia

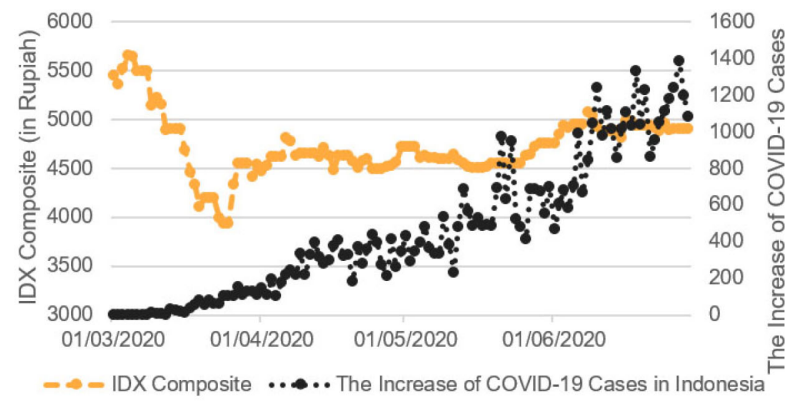

(b)

Fig. 1. USD/IDR exchange rate vs the increase of COVID-19 cases time series plot (a) and IDX composite vs The Increase of COVID-19 Cases time series plot (b). It can be seen that as the number of COVID-19 cases in Indonesia increases, both the USD/IDR exchange rate and the IDX composite are weakening.

Then suppose $k=1$ and $m=1$ (SISO), then the VARMAX Model $(p, q, r)$ can be stated as:

$$
\begin{aligned}
\phi_{p}(B) Y_{t} & =\gamma_{r}(B) X_{t}+\theta_{q}(B) a_{t} \\
Y_{t} & =\frac{\gamma_{r}(B)}{\phi_{p}(B)} X_{t}+\frac{\theta_{q}(B)}{\phi_{p}(B)} a_{t}
\end{aligned}
$$

Note that Eq. (5) is similar to the Transfer Function Model equation which has the order of the transfer function $(b, r, s)$ and the order

$$
\begin{aligned}
Y_{t} & =\frac{\omega(B) B^{b}}{\phi_{p}(B)} X_{t}+\frac{\theta_{q}(B)}{\phi_{p}(B)} a_{t} \\
& =v(B) X_{t}+\frac{\theta_{q}(B)}{\phi_{p}(B)} a_{t}
\end{aligned}
$$

Therefore, the univariate VARMAX Model $(p, q, r)$ in Eq. (5) will be the same as the Transfer Function Model when $\frac{\gamma_{r}(B)}{\phi_{p}(B)}=v(B)$ or $\gamma_{r}(B)=v(B) \phi_{p}(B)$. The parameters of this model can be estimated by minimizing the sum square of errors through multivariate generalized least square method. The sum square of errors is defined by utilizing matrix operations that include vectorization and kronecker multiplication (Tsay, 2014).

\section{Data analysis}

\subsection{Data analysis using the VARMAX model}

In this section, the impact of COVID-19 cases in Indonesia is used to forecast the USD/IDR exchange rate and the IDX Composite. Suppose daily data of USD/IDR $\left\{Y_{1, t}\right\}$ and IDX Composite $\left\{Y_{2, t}\right\}$. These two series can be influenced by the increase in the number of COVID-19 cases in Indonesia $\left\{X_{t}\right\}$. This paper compares which method is more accurate to forecast the USD/IDR exchange rate and IDX Composite. Previously, here are the characteristics of the data. Suppose $\boldsymbol{Y}_{t}=\left(Y_{1, t}, Y_{2, t}\right)^{\prime}, X_{t}=\left(X_{t}\right)^{\prime}, k=2$, and $m=1$ (SIMO). In this paper, the data for the period 1 March 2020-29 June 2020 is used as in-sample data while the data in period 30 June 2020-3 July 2020 is used as out-sample data. The two endogenous variables have a value of 0 on every Saturday and Sunday since the market is closed on that day. As a consequence, for Friday data will be also used for both Saturday data and Sunday data.

According to Table 1, it is known that the lowest USD/IDR exchange rate is Rp 13,085.40. This means that the strongest appreciation of the Rupiah is Rp 13,085.40 while the Rupiah depreciates the strongest at the level of Rp 16,504.80, namely on 24 March 2020 while the increase in the number of COVID-19 cases in Indonesia jumped by 107 cases. As the COVID-19 case in Indonesia has increased, investors have attracted their Rupiah to buy US 
Table 1

Descriptive statistics of endogenous variables and exogenous variables

\begin{tabular}{cccccc}
\hline Variables & $\mathrm{T}$ & Mean & Std. deviation & Minimum & Maximum \\
\hline$Y_{1, t}$ & 121 & 14995 & 808.31 & 13085.4 & 16504 \\
$Y_{2, t}$ & 121 & 4725.1 & 314.7 & 3937.6 & 5650.1 \\
$X_{t}$ & 121 & 455.3 & 376.2 & 0 & 1385 \\
\hline
\end{tabular}

Table 2

Decision of the ADF test results. The USD/IDR exchange rate and the increase of COVID-19 cases are not stationary

\begin{tabular}{ccc}
\hline Variables & $p$-value & Decision \\
\hline$Y_{1, t}$ & 0.39 & Data is not stationary \\
$Y_{2, t}$ & 0.02 & Data is stationary \\
$X_{t}$ & 0.11 & Data is not stationary \\
\hline
\end{tabular}

Table 3

Sample correlation matrix function table for endogenous variable: (a) Before and (b) After differencing

\begin{tabular}{|c|c|c|c|c|c|c|c|c|c|c|c|c|c|}
\hline \multicolumn{14}{|c|}{ Schematic representation of cross correlations } \\
\hline \multicolumn{14}{|c|}{ (a) Before differencing } \\
\hline Variable/lag & 0 & 1 & 2 & 3 & 4 & 5 & 6 & 7 & 8 & 9 & 10 & 11 & 12 \\
\hline$Y_{1, t}$ & +- & +- & +- & +- & +- & +- & +- & +- & +- & +- & +- & +- & +- \\
\hline$Y_{2, t}$ & -+ & -+ & -+ & -+ & -+ & -+ & -+ & -+ & -+ & -+ & - & - & - . \\
\hline \multicolumn{14}{|c|}{ (b) After differencing } \\
\hline Variable/lag & 0 & 1 & 2 & 3 & 4 & 5 & 6 & 7 & 8 & 9 & 10 & 11 & 12 \\
\hline$Y_{1, t}$ & + . & .. & .. & .. & .. & .. & - . & .. & .. & .. &.. &.. &.. \\
\hline$Y_{2, t}$ &.+ &.+ &.+ &.+ &.+ &.+ &.+ &.+ &.+ & .. & .. & .. &.. \\
\hline
\end{tabular}

dollars, as a result, the value of the Rupiah has weakened. The average USD/IDR exchange rate is Rp 14,995.31 with a standard deviation of 808.31 .

The average IDX Composite value is Rp 4,725.1 and the highest value of the IDX Composite is Rp 5,650.14 meaning the strongest IDX Composite value is Rp 5,650.14 at 4 March 2020. However, after the strengthening of the IDX Composite on that date, the value of the IDX Composite continues to decline to below Rp 5,000.00 as cases of COVID-19 in Indonesia increase. It is also similar to the USD/IDR exchange rate, investors choose to invest in assets that are safer than placing their assets in shares in Indonesia, causing IDX Composite to touch the lowest level in history. It can be seen that, with the increase in COVID-19 cases in Indonesia, investors are shifting their assets to a safe haven, one of which is US dollars. The following is the movement of the two variables data displayed in the form of a time series plot.

The use of graphics in detecting that a series is stationary needs foresight so that the data stationarity test is performed using the Augmented Dickey-Fuller Test (ADF Test) with null hypotheses is "Data is not stationary" with alternative hypotheses is "Data is stationary". The results are summarized in Table 2.

According to Table 2, the USD/IDR exchange rate and the increase of COVID-19 cases both are not stationary for $\alpha=0.05$. As a result, these data need to be differentiated first. In the formation of the VARMAX Model, it is necessary to fulfill the basic assumption, namely stationarity. The next step is to look at the Sample Cross Correlation Matrix Function table and the Partial Autoregression Matrix Function table:

Based on Table 3(a), it can be seen that the (+) sign and the (-) sign that appear simultaneously in each lag shows that the data is not stationary. Then a similar pattern is seen for the two endogenous variables, so it needs to be differentiated for both variables and the Sample Correlation Matrix Function as obtained on Table 3(b).

It can be seen that the number of the (+) signs that appear simultaneously for the two endogenous variable in Sample Correlation Matrix Function Table is significant at lag 0 . The $(+)$ sign denotes a value that is more than 2 times the standard error by observing 121 data, the (-) sign denotes a value less than 2 times the standard error by observing 121 data, while the (.) sign denotes a value that is at the interval -2 times the standard error up to 2 times the standard error which can be calculated as:

$$
2 \times S E\left(\rho_{i j}(1)\right)=2 \times \sqrt{\frac{1}{121-1}}
$$


Table 4

Partial autoregression matrix function table for endogenous variable after differencing

\begin{tabular}{cccccccccccccc}
\hline \multicolumn{11}{c}{ Schematic representation of partial autoregression } \\
\hline Variable/Lag & 1 & 2 & 3 & 4 & 5 & 6 & 7 & 8 & 9 & 10 & 11 & 12 \\
\hline$Y_{1, t}$ &.. &.. &.. &.. &.. &.. &.- &.. &.. &.. &.. &.. \\
$Y_{2, t}$ &.+ &.- &.. &.. &.. &.. &.. &.. &.. &.. &.. &.- \\
\hline
\end{tabular}

Table 5

Minimum information criterion based on AICC. The possible models are AR(1) and MA(1)

\begin{tabular}{ccccc}
\hline \multicolumn{5}{c}{ Minimum information criterion } \\
\hline Lag & MA0 & MA1 & MA2 & MA3 \\
\hline AR0 & 22.48 & 22.04 & 21.89 & 21.68 \\
AR1 & 20.13 & 20.05 & 20.12 & 20.12 \\
AR2 & 20.11 & 20.11 & 20.12 & 20.14 \\
AR1 & 20.15 & 20.16 & 20.16 & 20.15 \\
\hline
\end{tabular}

$=2 \times \sqrt{0.01}$

$=0.18$

It can be said that the $(+)$ sign is greater than 0.18 , which means there is a correlation between each variable with the variable shown in lag. Furthermore, it can be seen in the Partial Autoregression Matrix Function Table as follows:

It can be said that the value is between \pm 0.18 which means there is no correlation between each variable with the variable shown in lag. The number of $(+)$ signs that appear simultaneously in the Partial Autoregression Matrix Function Table is significant in the first lag. Order selection is also considered through the smallest AICC information on Minimum Information Criterion as follows:

Based on Table 5, model candidate can be chosen based on the smallest AICC value, namely VARMAX $(1,1,1)$ Model with a value of 20.05. After obtaining the desired VARMAX Model, the next step is to estimate all the model parameters as follows:

$$
\begin{aligned}
\left(\begin{array}{l}
Y_{1, t} \\
Y_{2, t}
\end{array}\right)= & \left(\begin{array}{c}
3154.92 \\
66.47
\end{array}\right)+\left(\begin{array}{c}
0.08 \\
-0.003
\end{array}\right) X_{t}+\left(\begin{array}{c}
-0.23 \\
0.007
\end{array}\right) X_{t-1}+\left[\begin{array}{cc}
0.87 & -0.23 \\
0.007 & 0.96
\end{array}\right]\left(\begin{array}{l}
Y_{1, t-1} \\
Y_{2, t-1}
\end{array}\right) \\
& +\left[\begin{array}{cc}
0.11 & 0.33 \\
0.04 & -0.14
\end{array}\right]\left(\begin{array}{l}
e_{1, t-1} \\
e_{2, t-1}
\end{array}\right)
\end{aligned}
$$

The above equation can be formed in a mathematical equation:

1. For the USD/IDR exchange rate:

$$
\hat{Y}_{1, t}=3154.92+0.08 X_{t}-0.23 X_{t-1}+0.87 Y_{1, t-1}-0.23 Y_{2, t-1}+0.11 e_{1, t-1}+0.33 e_{2, t-1}
$$

2. For IDX composite:

$$
\hat{Y}_{2, t}=66.47-0.003 X_{t}+0.007 X_{t-1}+0.007 Y_{1, t-1}+0.96 Y_{2, t-1}+0,04 e_{1, t-1}-0.14 e_{2, t-1}
$$

Since the unit of the response variables (USD/IDR exchange rate and IDX composite) are in IDR meanwhile the predictors (right-side of the equation) have various units, then the parameters coefficient will take the roles to adjust the unit. For example, the estimated parameter $\hat{\gamma}_{0,1}=0.08$ and $\hat{\gamma}_{0,2}=-0.003$ for number of COVID-19 cases $\left(X_{t}\right.$, in person unit) has unit is IDR/person. The same way applies to other models. Notice that for both USD/IDR exchange rate and IDX composite are influenced by the common predictors with opposite sign, except for the one-step-behind of USD/IDR exchange rate, which both have positive sign. It means that the USD/IDR exchange rate in previous day will strengthen the current's rate in the same direction (positive coefficient).

After the VARMAX Model is selected, it will be checked whether the model is feasible or not to be predicted using the diagnostic test and the multivariate normal distribution at the residual.

1. The Portmenteau test

The Portmenteau test is used to test whether the residuals are identical and independent, using the following hypotheses formulation:

$H_{0}$ : Residual vector is white noise. 
Table 6

Schematic representation of cross correlation of residuals table. Correlation only occurs at lag 0

\begin{tabular}{cccccccccccccccc}
\hline \multicolumn{10}{c}{ Schematic representation of cross correlations of residuals } \\
\hline Variable/Lag & 0 & 1 & 2 & 3 & 4 & 5 & 6 & 7 & 8 & 9 & 10 & 11 & 12 \\
\hline$Y_{1, t}$ &.+ &.. &.. &.. &.. &. &.. &.. &.. &.. &.. &.. &.. \\
$Y_{2, t}$ &.+ &.. &.. &.. &.. &.. &.. &.. &.. &.. &.. &.. &.. \\
\hline
\end{tabular}

Table 7

The actual and prediction for the next 4 days along with the Upper Limit (UL) and Lower Limit (LL) with a 95\% confidence interval for (a) USD/IDR exchange rate and (b) IDX composite

\begin{tabular}{cccccc}
\hline Date of Y.2020 & $Y_{1,121}(l)$ & $\hat{Y}_{1,121}(l)$ & $95 \% \mathrm{LL}$ & 95\% UL & $\frac{\left|\hat{Y}_{1,121}(l)-Y_{1,121+l}\right|}{Y_{1,121+l}}$ \\
\hline \multicolumn{5}{c}{ (a) The USD/IDR exchange rate } \\
\hline 30 June $(l=1)$ & 14470 & 14178.39 & 13727.39 & 14629.39 & 0.020 \\
1 July $(l=2)$ & 14194.1 & 14143.66 & 13568.81 & 14718.51 & 0.003 \\
2 July $(l=3)$ & 14187.9 & 14121.23 & 13456.87 & 14785.59 & 0.004 \\
3 July $(l=4)$ & 14298.8 & 14109.07 & 13371.88 & 14846.25 & 0.010 \\
\hline \multicolumn{5}{c}{ (b) The IDX Composite } \\
\hline 30 June $(l=1)$ & 4950.39 & 4900.32 & 4717.94 & 5082.69 & 0.001 \\
1 July $(l=2)$ & 4914.39 & 4895.44 & 4623.79 & 5167.07 & 0.003 \\
2 July $(l=3)$ & 4966.78 & 4890.34 & 4556.90 & 5223.78 & 0.02 \\
3 July $(l=4)$ & 4973.79 & 4885.14 & 4503.55 & 5266.73 & 0.02 \\
\hline
\end{tabular}

$H_{1}$ : Residual vector is not white noise.

Take a look at the schematic representation of cross correlation of residuals on Table 6. It shows that the correlation only occurs at lag 0 . It is supported by the Portmenteau test result which is for all lag times of the VARMAX $(1,1,1)$ model's residuals have $p$-values which are greater than $1 \% \leqslant \alpha<10 \%$. Then $H_{0}$ is not rejected, which means residual vector is white noise. Thus, it can be said that the residual is identical and independent (white noise).

2. The multivariate normal residual assumptions test.

The last stage of the diagnostic test is testing the multivariate normal distribution using the null hypotheses is "residual vector is multivariate normal distribution". Using the chi-square test, the obtained $p$-values are less than 0.001 for both residuals of $Y_{1, t}$ and $Y_{2, t}$.

Since the $p$-values are less than $1 \% \leqslant \alpha<10 \%$, then $H_{0}$ is not rejected. It can be concluded that both residuals are not multivariate normal distribution. It might be due to the intervention and outlier factors.

After obtaining the VARMAX model, then the next process is to predict USD/IDR exchange rate and the IDX Composite using the VARMAX $(1,1,1)$ model for some time to come with $T=121$ observations that have been known by the realization value of $\hat{Y}_{1,121}(l)$ and $\hat{Y}_{2,121}(l)$. The prediction of the USD/IDR exchange rate and the IDX composite will be carried out for the next 4 steps, namely 30 June-3 July 2020 are summarized in Table 7.

Based on Table 7(a) and (b), the prediction results for both USD/IDR exchange rate and IDX composite are still between the respective 95\% lower limit and the 95\% upper limit. The results of the absolute difference between the predicted value and the actual value divided by the actual value quite small for the next 4 days. This shows that the VARMAX $(1,1,1)$ model for predicting the USD/IDR exchange rate and IDX composite is equally good. Likewise in Fig. $2 \mathrm{a}$ and $\mathrm{b}$, the respective comparison plot looks quite close in value. In addition, its MAPE value is very good at $1.04 \%$ and $0.95 \%$ respectively. The predictive ability for the VARMAX $(1,1,1)$ model is very good since the MAPE value is less than $10 \%$.

\subsection{Data analysis using the transfer function model}

An analysis of the impact of COVID-19 cases in Indonesia on the USD/IDR exchange rate using the transfer function model is being conducted here. Using the characteristic from the previous study above, the next step is to identify the ARIMA Model for the exogenous variable, namely, the increase of the number of COVID-19 cases in 
The USD/IDR Exchange Rate Comparison Plot

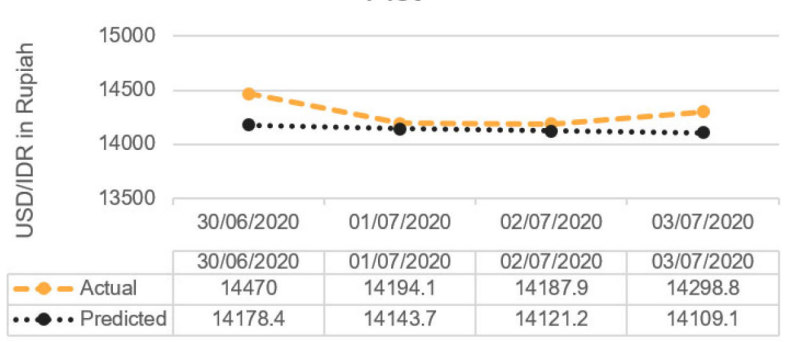

- - Actual $\ldots \bullet .$. Predicted

(a)
The IDX Composite Comparison Plot

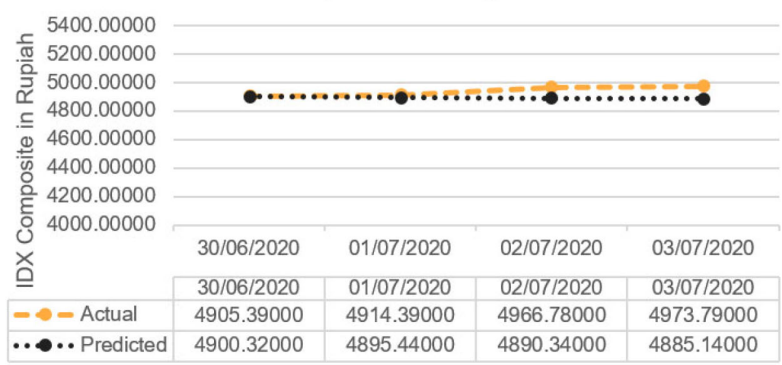

$-\bullet-$ Actual $\ldots \bullet$... Predicted

(b)

Fig. 2. Comparison of Predicted Value and Actual Value for (a) The USD/IDR exchange rate, and (b) The IDX composite prediction. Both plots show the quite close of predicted and actual values.

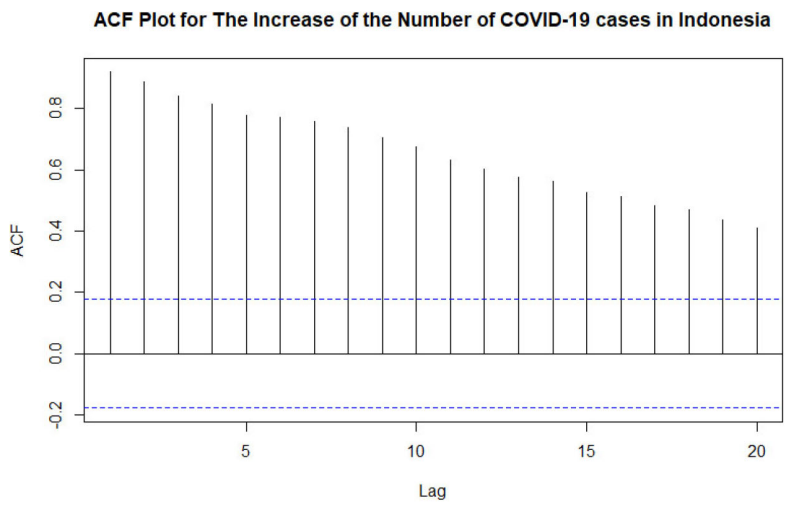

(a)

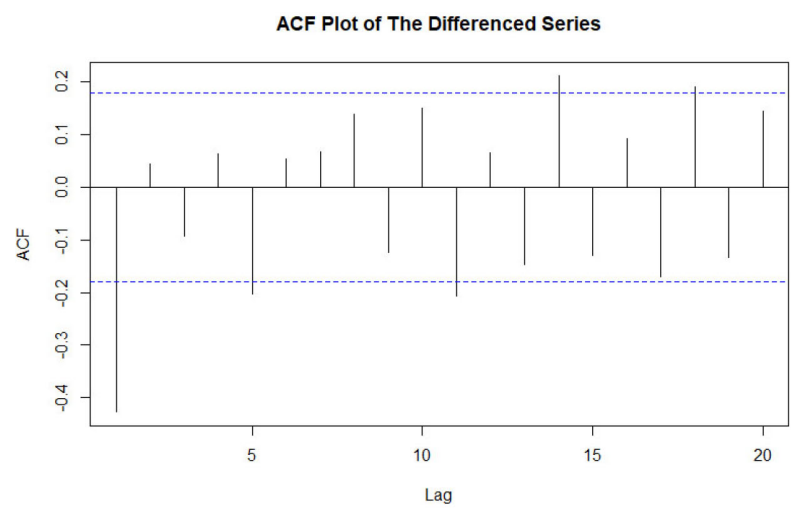

(c)

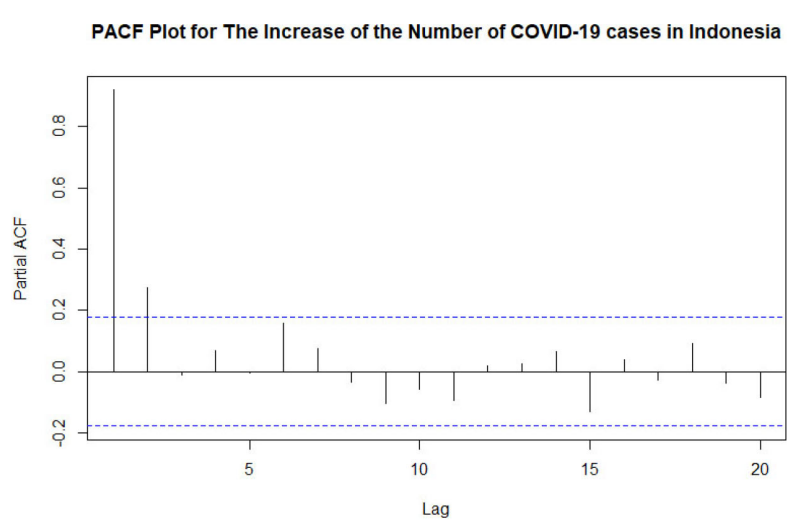

(b)

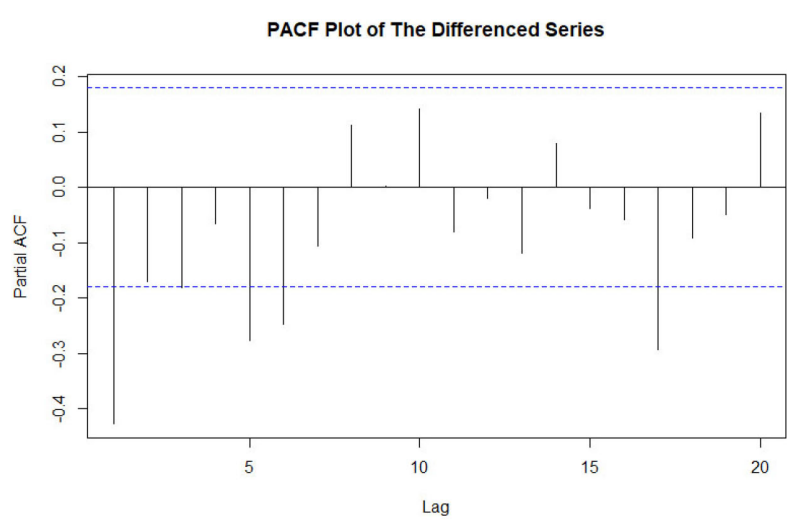

(d)

Fig. 3. ACF and PACF Plot for the Exogenous Variable: (a) ACF and (b) PACF of original series, (c) ACF and (b) PACF of differenced series. Those plots are used to identify the suitable ARIMA Model.

Indonesia. In the formation of the transfer function mModel, the basic assumption is stationary. Take a look at the ACF and PACF plots of the increase of the number of COVID-19 cases in Indonesia on the following Fig. 3.

The two figures above show that the data is not stationary in the average since in Fig. 3a shows exponential decay. Therefore, to make the data is stationary in the average, it needs to be differenced first whose ACF and PACF are given by (c) and (d). From these figures, the ARIMA model can be determined based on the significant lag in the 
Table 8

The significance test of the todel parameters

\begin{tabular}{ccccc}
\hline Variable & Parameter & Estimate & $p$-value & AIC \\
\hline ARIMA $(1,1,1)$ & $\phi_{1}$ & 0.09 & 0.49 & 1488.69 \\
& $\theta_{1}$ & -0.65 & $<0.001$ & \\
ARIMA $(0,1,1)$ & $\theta_{1}$ & -0.59 & $<0.001$ & 1487.12 \\
\hline
\end{tabular}

Normal Q-Q Plot

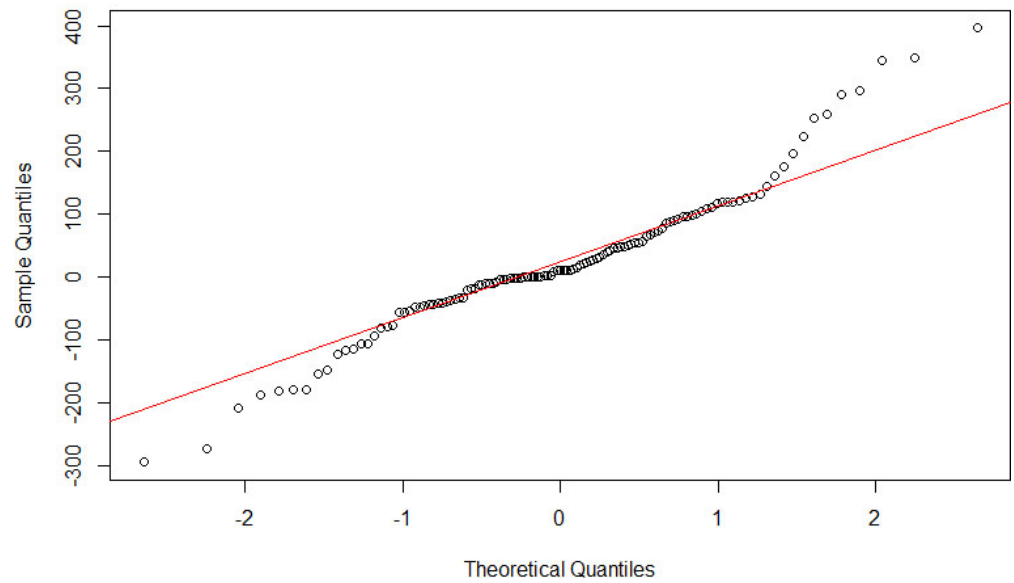

Fig. 4. QQ-plot of residual ARIMA $(0,1,1)$ model. It can be seen that the ARIMA $(0,1,1)$ model meets the normality assumption because of the large amount of data values are along the lines.

ACF and PACF plots. The ACF and PACF cut off at the first lag, thus the possible ARIMA Model for the exogenous variable are the $\operatorname{ARIMA}(1,1,1)$ model and the $\operatorname{ARIMA}(0,1,1)$ model.

The next step is the estimation and evaluation of both models parameters. To test the parameters significance, the null hypotheses is "exogenous variable is not significant" versus "exogenous variable is significant". The estimators and its significance are presented on Table 8. In Table 8, parameter significance test has been performed for two possible models. The number in bold indicates that the parameter has $p$-value is more than $1 \% \leqslant \alpha \leqslant 5 \%$, so the parameter is not significant in the model. Note that the $\operatorname{ARIMA}(1,1,1)$ model has one insignificant parameter and the AIC's model is 1488.69 . Then by eliminating the insignificant parameter from the previous model, the AIC value become 1487.12 which is smaller than the previous one. Since the ARIMA $(0,1,1)$ Model has a significant parameter and has the smallest AIC value, thus it is a suitable model for the exogenous variable.

After obtaining the suitable model, it is necessary to do a diagnostic test by conducting a white noise test and a residual normality test. The null hypotheses is "the residual is white noise" and vise versa for the alternative hypotheses. Based on the Ljung-Box test, it is found that only at 5 'th lag the $p$-value is greater than $1 \% \leqslant \alpha \leqslant 5 \%$, means that the null hypotheses is not rejected only at 5'th lag. As a result, the residual does not fulfill the white noise assumption.

Hereafter, the normality assumption of the $\operatorname{ARIMA}(0,1,1)$ residuals be checked through the QQ-Plot, as can be seen on Fig. 4. It shows that quite a lot of data values are along the normal line, so it can be said that the residuals are normally distributed.

Since by seeing the QQ-Plot is considered to be less objective, another test is carried out to check whether the residuals are normally distributed using the Jarque Bera test with the null hypothesis is "residual is not normally distributed". It is obtained that the $p$-value is less than $1 \% \leqslant \alpha \leqslant 5 \%$, means null hypotheses is rejected. Therefore, the residuals are normally distributed.

Based on the estimated parameter values in Table 8, the model can be written as:

$$
X_{t}=a_{t}-0.59 a_{t-1}
$$

The pre-whitening process for the exogenous series is

$$
\alpha_{t}=X_{t}+0.59 \alpha_{t-1}
$$




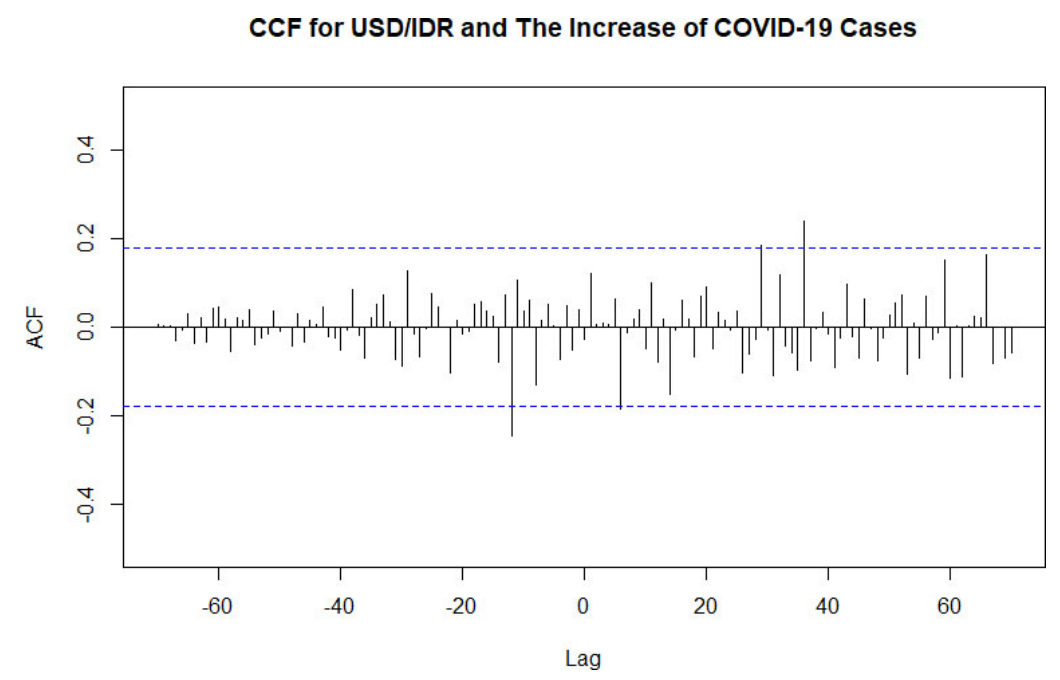

Fig. 5. CCF plot of the pre-whitened exogenous and pre-whitened endogenous variable. It can be seen that the main order of the transfer function is $(b=6, r=0, s=2)$.

such that the pre-whitening process for the output endogenous series is,

$$
\beta_{t}=Y_{t}+0.59 \beta_{t-1}
$$

The pre-whitening step in the exogenous series must also be executed in the endogenous series. This is for maintaining the integrity of the functional relationship of the transfer function maps $X_{t}$ into $Y_{t}$.

In order to obtain the transfer function model, the Crosscorrelation Function (CCF) should be investigated. The $\mathrm{CCF}$ is used to determine the value of the main order of the transfer function, namely order $(b, r, s)$. Before forming the CCF Plot, the standard deviations are calculated for $\left\{\hat{\alpha}_{t}\right\}$ and $\left\{\hat{\beta}_{t}\right\}$, which are 115.19 and 277.82 consecutively. The CCF Plot of the pre-whitened exogenous and pre-whitened endogenous variable is as shown on Fig. 5 below. Note in Fig. 5, the possible main order of the transfer function is $(b, r, s)=(6,0,2)$, then the transfer function for the exogenous variable is:

$$
v(B)=\left(\omega_{0}-\omega_{1} B-\omega_{2} B^{2}\right) B^{6}
$$

The estimated parameters of the transfer function with order $(6,0,2)$ are $\hat{\omega}_{0}=0.01, \hat{\omega}_{1}=-0.18$, and $\hat{\omega}_{2}=-0.26$. Thus, the initial transfer function can be formed as:

$$
Y_{t}=\left(\omega_{0}-\omega_{1} B-\omega_{2} B^{2}\right) X_{t-6}+N_{t}
$$

Next, the ARMA modeling will be performed on the noise series so that the white noise residual will be obtained. The ACF and PACF plot of residuals are presented in Fig. 6. Based on this plot, the possible model obtained is MA(1).

Thereafter, all parameters of the transfer function model are estimated and obtained that $\hat{\theta_{1}}=0.09, \hat{\omega}_{0}=0.003$, $\hat{\omega}_{1}=0.17$, and $\hat{\omega}_{2}=-0.24$. Thus, the final transfer function model can be formed as:

$$
\hat{Y}_{1, t}=\left(0.003+0.17 B+0.24 B^{2}\right) X_{t-6}+(1-0.09 B) a_{t}
$$

and be simplified into the following form:

$$
\hat{Y}_{1, t}=0.003 X_{t-6}+0.17 X_{t-7}+0.24 X_{t-8}+a_{t}-0.09 a_{t-1}
$$

After obtaining the transfer function model, it is necessary to do a diagnostic test between $\left\{\hat{a}_{t}\right\}$ and $\left\{\alpha_{t}\right\}$. The first is autocorrelation test with null hypotheses " $\left\{\hat{a}_{t}\right\}$ is not significantly correlated". By using Ljung-Box test, it is obtained that for all lag times, the $p$-values are greater than $1 \% \leqslant \alpha \leqslant 10 \%$. It means that do not reject the null hypotheses. Thus the residuals meet the white noise assumption.

Further, the crosscorrelation test is carried out using the null hypotheses is " $\left\{\hat{a}_{t}\right\}$ and $\left\{\alpha_{t}\right\}$ are not independent". By still using Ljung-Box test, it is obtained that all $p$-values are greater than $1 \% \leqslant \alpha \leqslant 10 \%$, so null hypotheses is 
Table 9

The USD/IDR exchange rate prediction for the next 4 days along with the upper and lower limits with a 95\% confidence limits

\begin{tabular}{|c|c|c|c|c|c|}
\hline Date of Y.2020 & $Y_{1,121+l}$ & $\hat{Y}_{1,121}(l)$ & $95 \% \mathrm{LL}$ & $95 \% \mathrm{UL}$ & $\frac{\left|\hat{Y}_{1,121}(l)-Y_{1,121+l}\right|}{Y_{1,121+l}}$ \\
\hline 30 June $(l=1)$ & 14470 & 14260.30 & 13783.08 & 14737.58 & 0.01 \\
\hline 1 July $(l=2)$ & 14194.1 & 14294.60 & 13651.19 & 14937.98 & 0.007 \\
\hline 2 July $(l=3)$ & 14187.9 & 14320.79 & 13546.10 & 15095.48 & 0.009 \\
\hline 3 July $(l=4)$ & 14298.8 & 14347.51 & 13460.75 & 15234.26 & 0.003 \\
\hline
\end{tabular}
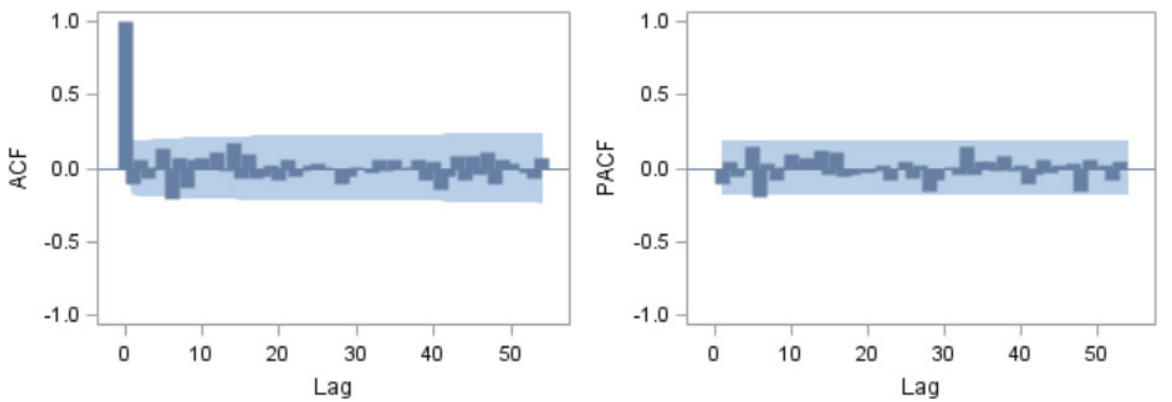

Fig. 6. ACF dan PACF plot of the residual. The identified time series model from the ACF and PACF plot is MA(1).
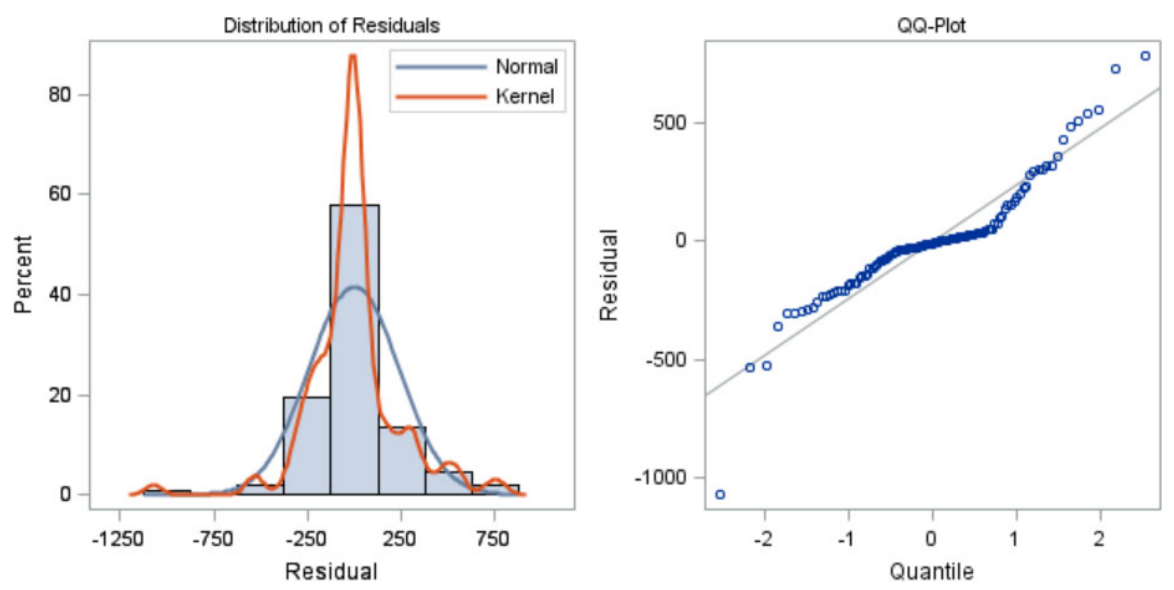

Fig. 7. The residual normality test. The graph shows that the residuals are normally distributed.

not rejected. Therefore, residuals are independent. In addition to the diagnostic test, the residual normality can also be tested through the following QQ-Plot on Fig. 7.

Figure 7 shows that quite a lot of data values are along the normal line, so it can be said that the residuals are normally distributed. Thus, the next step is to predict the USD/IDR exchange rate with $T=121$ observations that have been known with the realization value is $\hat{Y}_{121}(l)$. Table 9 shows the USD/IDR exchange rate prediction will be carried out for the next 4 days, namely on 30 June 2020-3 July 2020.

Based on Table 9, the prediction results are still between the $95 \%$ lower limit and the $95 \%$ upper limit and also the results of the absolute difference between the predicted value and the actual value divided by the actual value quite small for the next 4 days. This shows that the transfer function model for predicting the USD/IDR exchange rate is quite good. Likewise in Fig. 8, the comparison plot looks quite close in value. In addition, its MAPE value is very good at $0.86 \%$. The predictive ability for the transfer function model is very good since the MAPE value is lower $10 \%$. 
The USD/IDR Exchange Rate Comparison Plot

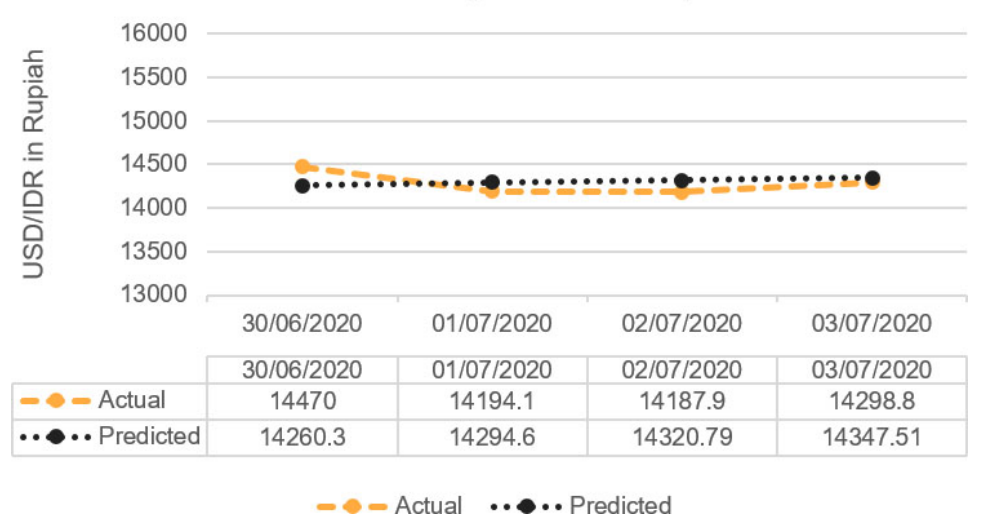

Fig. 8. Comparison of predicted value and actual value. The USD/IDR exchange rate prediction are quite close from the actual value.

\section{Discussion}

On Section 3, two case studies were conducted using different approaches. First, the VARMAX model is used to model the prediction of the USD/IDR exchange rate and the IDX composite which are influenced by the increase of the number of COVID-19 cases in Indonesia. Here, prediction of the USD/IDR is not only be influenced by the increase of the number of COVID-19 cases in Indonesia, but also by the IDX composite. Here are the final model:

1. For the USD/IDR exchange rate:

$$
\hat{Y}_{1, t}=3154.92+0.08 X_{t}-0.23 X_{t-1}+0.87 Y_{1, t-1}-0.23 Y_{2, t-1}+0.11 e_{1, t-1}+0.33 e_{2, t-1}
$$

This means that intercept will make the USD/IDR exchange rate today depreciate at Rp 3154.92. Then an increase of $1 \%$ in the number of COVID-19 cases in Indonesia will weaken the USD/IDR exchange rate by $0.08 \%$. A $1 \%$ increase in the yesterday's number of COVID-19 cases in Indonesia will strengthen the USD/IDR exchange rate by $0.23 \%$. Furthermore, an increase of $1 \%$ in the yesterday's USD/IDR exchange rate will make the USD/IDR exchange rate depreciate today by $0.87 \%$, while an increase of $1 \%$ in the yesterday's IDX Composite will make the USD/IDR exchange rate appreciate today by $0.23 \%$.

2. For IDX composite:

$$
\hat{Y}_{2, t}=66.47-0.003 X_{t}+0.007 X_{t-1}+0.007 Y_{1, t-1}+0.96 Y_{2, t-1}+0,04 e_{1, t-1}-0.14 e_{2, t-1}
$$

This means that the intercept will make the IDX composite increase by Rp 66.47 today. Then an increase of $1 \%$ in the number of COVID-19 cases in Indonesia will make the IDX composite today decrease by $0.003 \%$. An increase of $1 \%$ in the yesterday's number of COVID-19 cases in Indonesia will make the IDX composite today increase by $0.007 \%$. Furthermore, an increase of $1 \%$ in the yesterday's USD/IDR exchange rate will make the IDX composite increase today by $0.007 \%$, while an increase of $1 \%$ in the yesterday's IDX Composite will make the IDX composite today increase by $0.96 \%$.

From diagnostic checking of residuals, the multivariate normal assumption could not be fulfilled. It becomes the weakness of this model. When it is used for predicting, it is obtained that the MAPE value for the USD/IDR exchange rate is $1.04 \%$ which means that the model has a great predictive ability. As a matter of fact, from the comparison plot, it can be seen that the prediction for 1 July 2020 and 2 July 2020 is pretty close with the actual values.

Second, the transfer function model is used to model the prediction of the USD/IDR exchange rate. In this case, it was assumed that the USD/IDR exchange rate only be influenced by the increase of the number of COVID-19 cases in Indonesia. The final model is:

$$
\hat{Y}_{1, t}=0.003 X_{t-6}+0.17 X_{t-7}+0.24 X_{t-8}+a_{t}-0.09 a_{t-1}
$$

This means that an increase of $1 \%$ in the number of COVID-19 cases in Indonesia six days ago will weaken the USD/IDR exchange rate by $0.003 \%$. A $1 \%$ increase in the number of COVID-19 cases in Indonesia seven days ago 
will also weaken the USD/IDR exchange rate by $0.17 \%$. Furthermore, an increase of $1 \%$ in the number of COVID-19 cases in Indonesia eight days ago will make the USD/IDR exchange rate today depreciate by $0.24 \%$.

As consequence, the MAPE value for the USD/IDR exchange rate is $0.86 \%$ which means that the model has a great predictive ability. In fact, from the comparison plot, it can be seen that the last three predicted values are very close to the actual values. Based on these results, the USD/IDR exchange rate is better to be predicted using the transfer function model since it has lower MAPE value than using the VARMAX Model.

\section{Conclusion and remark}

According to Section 3, it can be concluded that the increase of the number of COVID-19 cases in Indonesia significantly affected the USD/IDR exchange rate. The findings in detail can be explained as follows:

1. The higher the increase of the number of COVID-19 cases in Indonesia will have an impact on the increasingly depreciating value of the USD/IDR exchange rate.

2. An increase of $1 \%$ in the number of COVID-19 cases in Indonesia six days ago will weaken the USD/IDR exchange rate by $0.003 \%$.

3. An increase of $1 \%$ in the number of COVID-19 cases in Indonesia seven days ago will weaken the USD/IDR exchange rate by $0.17 \%$.

4. An increase of $1 \%$ in the number of COVID-19 cases in Indonesia eight days ago will weaken the USD/IDR exchange rate by $0.24 \%$.

\section{Acknowledgments}

The authors thank to the RISTEK/BRIN grant, "Penelitian Dasar Unggulan Perguruan Tinggi (PDUPT)", 20192021 for supporting funds. Our gratitude for the reviewers of this manuscript.

\section{References}

Andayani, N., Sumertajaya, I. M., Ruchjana, B. N. \& Aidi, M. N. (2016). Comparison ARIMA-X and VARMA-X model to space time data: A case study of rice price in six provinces on java island, International Journal of Applied Mathematics and Statistics, 55(3).

Apriliandara, M., Suhartono, \& Prastyio, D. D. (2016). VARI-X model for currency inflow and outflow forecasting with Eid Fitr effect in Indonesia, AIP Conference Proceedings, 1746(1), 020041.

Box, G. E. P., Jenkins, G. M. \& Reisel, G. C., and Ljung, G. M. (2016). Time Series Analysis. Forecasting and Control, $5^{\text {th }}$ Ed. Wiley.

Cryer, J. \& dan Kung-Sik Chan D. (2008). Time Series Analysis: With Apllication in R Second Edition. Springer.

Ganegoda, N. C., Wijaya, K. P. \& Amadi, M., Erandi, K. K. W. H. \& Aldila, D. (2021). Interrelationship between daily COVID-19 cases and average temperature as well as relative humidity in Germany, Scientific Reports, 11(1).

Hasan M. B., Mahi, M., Sarker, T. \& Amin, M. R. (2021). Spillovers of the COVID-19 Pandemic: Impact on global economic activity, the stock market, and the energy sector, Journal of Risk and Financial Management, 14(5): 1-19.

Huda, N. M., Mukhaiyar. Forecasting dengue fever cases using autoregressive distributed lag model with outlier factor, AIP Conference Proceedings, 2268(1), 020005.

Liu, H., Zhu, L., Pan, Z., Ba, F., Liu, Y., Liu, Y., Patel, M., Farantatos, E. \& Bhatt, N. (2015). ARMAX-based transfer function model identification using wide-area measurement for adaptive and coordinated damping control, IEEE Transactions on Smart Grid, 8(3), 020005.

Lutkepohl, H. (2005). New Introduction to Multiple Time Series Analysis. Springer.

Masteriana, D., Riani, M. I. \& Mukhaiyar, U. (2019). Generalized STAR $(1 ; 1)$ model with outlier-case study of begal in Medan, North Sumatera, Journal of Physics: Conference Series, 1245(1), 012046.

Mukhaiyar, U., Huda, N. M. \& Pasaribu, U. S. (2020). Analysis of generalized space time autoregressive with exogenous variable (GSTARX) model with outlier factor, Journal of Physics: Conference Series, 1496(1), 012004.

Mukhaiyar, U., Huda, N. M., Sari, K. N. \& Pasaribu, U. S. (2019). Modeling dengue fever cases by using GSTAR (1; 1) model with outlier factor, Journal of Physics: Conference Series, 1366(1), 01122.

Nicholson, W., Matteson, D. S. \& Bien, J. (2015). VARX-L: Structured regularization for large vector autoregressions with exogenous variables, International Journal of Forecasting, 33(3).

Pasaribu, U. S., Mukhaiyar, U., Huda, N. M., Sari, K. N. \& Indratno, S. W. (2021). Modelling COVID-19 growth cases of provinces in java Island by modified spatial weight matrix GSTAR through railroad passenger's mobility, Heliyon, 7(2), e0602.

Saikhu, A., Hudiyanti, C. V., Buliali, J. L. \& Hariadi, V. (2020). Predicting COVID-19 confirmed case in surabaya using autoregressive integrated moving average, bivariate and multivariate transfer function, IOP Conf. Series: Materials Science and Engineering, 1077(2021), 012055. 
Spliid, H. (1983). A fast estimation method for the vector autoregressive moving average model with exogenous variables. Technical University of Denmark Press.

Tsay, R. S. (2014). Multivariate time series analysis with R and financial applications. Wiley.

Wei, W. W. S. (2006). Time series analysis: Univariate and multivariate method. Pearson.

Wei, Y. (2019). Oil price shocks, economic policy uncertainty and China's trade: A quantitative structural analysis, The North American Journal of Economics and Finance, 48: 20-31.

Widyanti, D. (2020). Pemodelan regresi deret waktu univariat dan multivariat menggunakan model fungsi transfer dan vektor autoregressive moving average with exogenous regressors. Tugas Akhir Program Sarjana Matematika. Institut Teknologi Bandung.

Zhao, L., Mbachu, J., Liu, Z. \& Zhang, H. (2019). Transfer function analysis: Modelling residential building costs in new zealand by including the influences of house price and work volume, MDPI: Buildings, 9(152).

References from Sites (website): The USD/IDR exchange rate and the IDX Composite Data 1 March 2020-3 July 2020, data obtained through internet sites: https://finance.yahoo.com/quote/USDIDR\%3DX/history?period1=1551657600\&period2=1583798400\&interval=1d\& filter=history \&frequency=1d. 